\title{
Sound attenuation by barriers in underwater
}

\author{
Kazuoki Kuramoto,* Shizuma Yamaguchi,** Kensei Oimatsu,* \\ and Shin'ya Kuwahara* \\ * Maritime Safety Academy, \\ Wakaba-cho, Kure, 737 Japan \\ **Faculty of Engineering, Yamaguchi University, \\ Tokiwadai, Ube, 755 Japan
}

(Received 19 December 1992)

\begin{abstract}
Sound attenuation characteristics of barrier for underwater sound propagation was investigated through a reduced scale cistern experiment. From the observed data for a thin barrier, it was found that the sound attenuation characteristics in underwater is almost the same as that in the air, which has been obtained in the noise control engineering field. Next, a useful functional form was derived for predicting the sound attenuation in underwater and it's effectiveness was confirmed experimentally by applying it to the data measured in an actual sea area. Furthermore, a possibility for the prediction of sound attenuation value in the case of a finite wide barrier was considered as well. As a result, sound attenuation characteristics for the wide barrier were understood by a double diffraction process.
\end{abstract}

Keywords: Sound attenuation, Underwater sound, Diffraction by barrier, Maekawa's chart, Cistern experiment

PACS number: 43. 30. Es

\section{INTRODUCTION}

In recent years, marine sports in the shallow water area as skin diving or scuba diving are popularized and simultaneously marine accidents arise along with it. One of the most simple and effective ways for preventing the diving accidents is to transmit directly an underwater acoustic signal to divers. The use of an audio signal is very efficient since the divers usually have no communication apparatus. In such a case, it is fundamentally important to investigate in advance how the audio acoustic signal radiated from the sea surface is attenuated in underwater.

The underwater sound attenuation characteristics have already been considered by many investigators. In most of these studies, however, theoretical and/or experimental considerations have been confined to the ultrasonic acoustic wave in deep-sea area. ${ }^{1)}$ In a previous paper, the underwater attenuation characteristics have been discussed in the case of the shallow water area but only in the free sound field. ${ }^{2}$ When a sound insulation barrier like a protect wall exists during the sound propagation path, underwater sound signal seems to be diffracted by it. Therefore, it is indeed necessary and worthwhile to study the sound attenuation effects by barrier so as to design the barrier for screening the ship noise and to estimate the power of a signal source toward the divers.

From the above practical viewpoint, in this paper, the sound attenuation characteristics of the barrier for underwater sound propagation is experimentally investigated, by paying attention to the case that the barrier exists in the middle of sound propagation path.

First, single diffraction by a thin barrier is considered through a reduced scale cistern experiment. Next, a practical equation is derived for estimating the sound attenuation in underwater. Furthermore the validity of the above estimation method is confirmed by using the data observed in an actual 
shallow water area. Finally, we discuss a possibility for prediction of the attenuation in the case of a finite wide barrier.

\section{EXPERIMENTAL SITUATION IN CISTERN}

Figure 1 shows a plan of the experimental arrangement of the barrier, sound source and receivers in cistern. Measurements were carried out for two cases as follows:

(1) Single diffraction by a thin barrier. The barrier was a $1.5[\mathrm{~cm}]$ thick composite plate, which made from a $3[\mathrm{~mm}]$ aluminum thin plate covered both side with $6[\mathrm{~mm}]$ polyethylene mat to avoid the transmission waves through the barrier (Appendix). The sound source (denoted by $S_{1}$ in Fig. 1) locates at the same level with top of barrier to regard as the single diffraction process.

(2) Double diffraction by a wide barrier. A 3 [cm] thick plate of wood was used as a barrier. The position of the sound source (denoted by $S_{2}$ in Fig. 1) was $5[\mathrm{~cm}]$ inside from the level of the barrier edge.

In both cases, a bursted-tone of 5 waves (carrier frequency is 70,100 and $150 \mathrm{kHz}$ ) was used in order to avoid the multiple reflections in cistern. The receiver $\mathrm{R}$ was set up to obtain the reference waves simultaneously. Accordingly, distances of $\mathrm{S}_{1} \mathrm{R}$ and $S_{1} P$ are equal. Sound sources $S_{1}, S_{2}$ and receivers $P, R$ are fixed $15[\mathrm{~cm}]$ depth under the surface of water. The path length differences were changed variously by moving the two receivers $(\mathrm{P}, \mathrm{R})$ at the same time (described by arrows in Fig. 1). The path length differences were determined by measuring accurately the distance $y$ in Fig. 1.

\section{RESULTS AND DISCUSSION}

An example of the sound pressure waves observed at the receiver $\mathbf{R}$ and $\mathbf{P}$ is shown in Fig. 2. Two sound waves divided clearly can be recognized, that is, one propagated directly in underwater and the other reflected one time at the surface of water. Although some complex waves are observed with some delay time, we pay our attention to only the beginning two waves in the following.

3.1 Sound Attenuation Characteristics of Thin Barrier

In order to compare the underwater sound at-

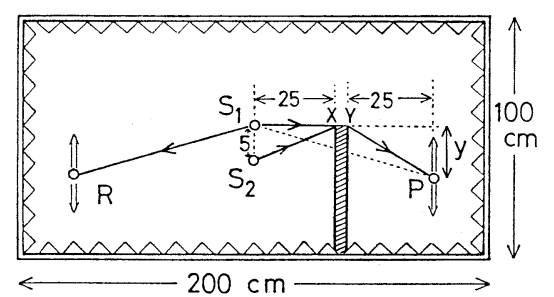

Fig. 1 The experimental situation for observing the sound attenuation effect.

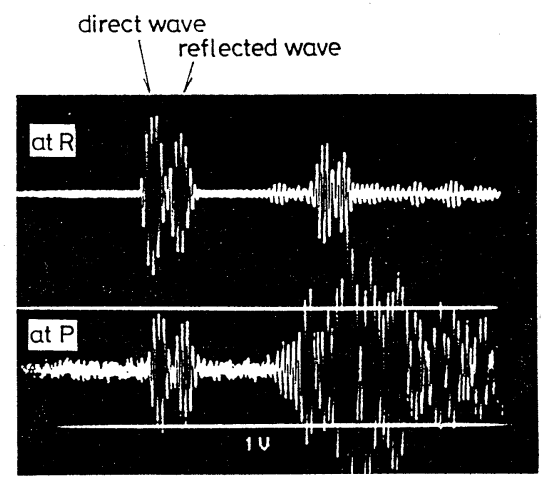

Fig. 2 Sound pressure waves received at two observation points of at $\mathrm{R}$ and at $\mathrm{P}$.

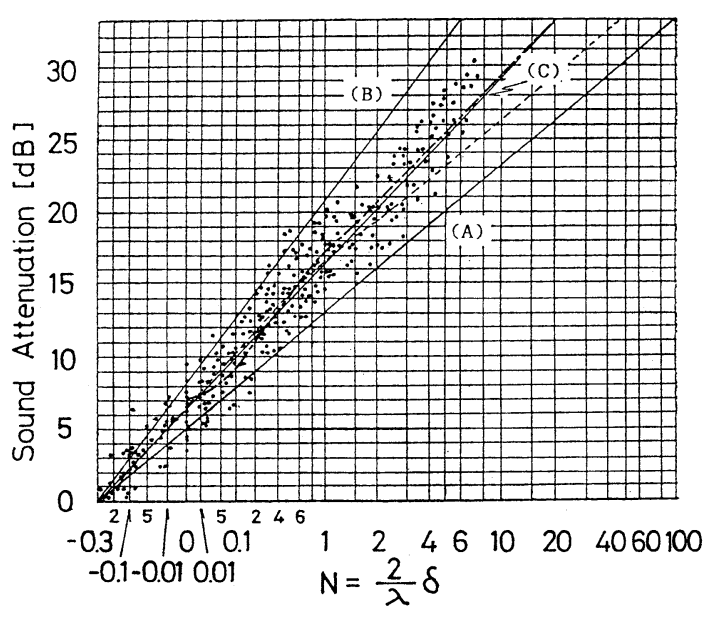

Fig. 3 Sound attenuation characteristics by a thin barrier for underwater sound propagation (summarized chart).

tenuation characteristics with those in air, all experimental data obtained in this study are plotted in the so-called Maekawa's chart, ${ }^{3)}$ which is very famous in the field of noise control engineering in 


\section{K. KURAMOTO et al.: SOUND ATTENUATION BY BARRIERS IN UNDERWATER}

the air. Figure 3 indicates the summarized chart where all experimental results have been plotted. As is well known, the vertical axis in the Maekawa's chart shows the attenuation of sound level at a receiver by the barrier in free space, and the horizontal axis the fresnel number $N$, which is the difference of sound propagation path length $\delta$ divided by a half wavelength $\lambda / 2$. It is necessary to pay our special attention that the horizontal axis is not a simple logarithmic scale and includes the negative region. Straight line (A) in Fig. 3 indicates the safe side of sound attenuation value in the air obtained experimentally by Dr. Maekawa from the viewpoint of the design of sound shielding barrier, and dotted line shows the sound attenuation value obtained theoretically by using the well-known Kirchhoff's approximations.

Experimental values in the present study exist between the lines (A) and (B). As far as we refer to the fact that the experimental values exist above the line (A), the sound attenuation characteristics in underwater is almost the same as in air. From the experimental results shown in Fig. 3, we can summarize as follows:

(1) In order to design the barrier for screening the underwater sound propagation such as a ship noise, the Maekawa's results (straight line (A) in Fig. 3) can be used as it was determined at a safe side.

(2) When we consider the acoustic signal transmission to divers, straight line (B) is adequate for estimating sound attenuations as it was drawn at a large attenuation side.

(3) For asking the sound attenuation itself, straight line $(\mathrm{C})$ which grasps outline of the attenuation characteristics in underwater should be employed.

In general, line (C) cannot be expressed in an exact functional form because the horizontal axis of Maekawa's chart is not simple scale. From a viewpoint of practical application, a suitable functional form is strongly required. By analogy of other investigations, ${ }^{4)}$ we introduce a function as

$$
\Delta L=a \log _{10}(N+0.2)+b, \quad(N \geqq 0) .
$$

Hereupon, $\Delta L$ denotes the difference of sound level at receiver between the barrier exists or not in free space. The values of two parameters, $a$ and $b$, in Eq. (1) were determined to be 13.1 and 16.3 , respectively, by using the least squares method. Dashdot line in Fig. 3 is the calculated curve from

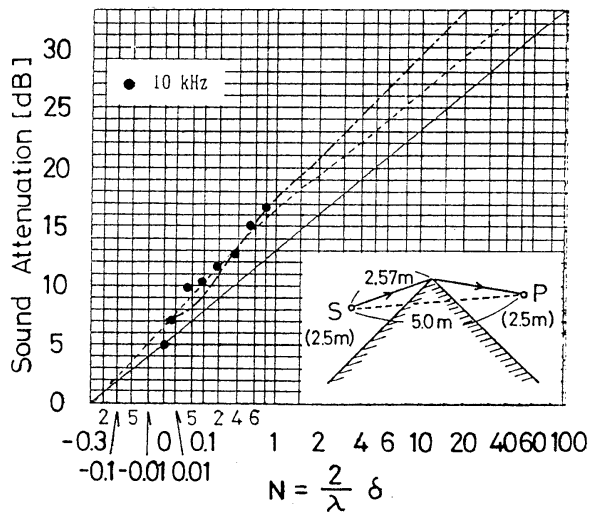

Fig. 4 A comparison between the sound attenuation curve estimated by using Eq. (1) and observed values measured in an actual sea area.

Eq. (1) with the parameters obtained above in the region $N \geqq 0$.

In order to confirm the validity of Eq. (1), acoustic measurements were made in the actual sea area. Experiments were carried out at the corner of a reclaimed ground in the Hiroshima Bay. The experimental conditions on geometry is indicated in inset of Fig. 4. The sound source and receivers were set up $2.5[\mathrm{~m}]$ depth under the water by hanging from the long poles, respectively. The frequency of sound signal was $10[\mathrm{kHz}]$. Experimental results obtained at several propagation path difference are shown in Fig. 4. It can be found that the dash-dot curve grasps roughly the tendency of experimental points though the number of data is not many. This fact shows that Eq. (1) is also fully useful for estimating the sound attenuations in actual sea area.

\subsection{Sound Attenuation Characteristics of Wide Barrier}

In the case of a finite wide barrier, it is impossible to explain the experimental results by a single diffraction process because the sound diffracts twice at the corners of the barrier during propagation. Then, we attempt to evaluate the data by means of a double diffraction process, with an assumption that the diffraction for wide barrier can be divided to two singles. Accordingly, the following equation, which is usually employed in air, ${ }^{5)}$ was used:

$$
\Delta L=\Delta L_{1}+\Delta L_{2}+20 \log _{10}\left(r^{\prime} / r\right)-7,
$$

where $\Delta L_{1}$ and $\Delta L_{2}$ are the sound attenuations in 


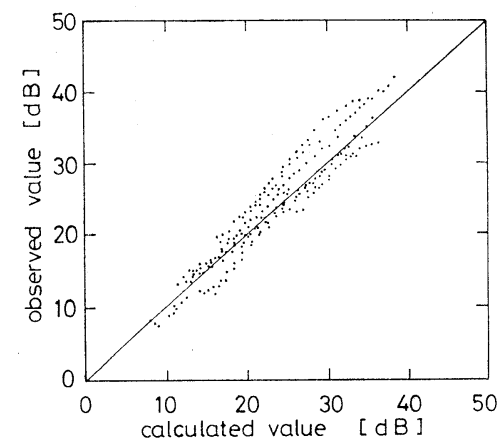

Fig. 5 A comparison between the theoretical and experimental values for the sound attenuation in the case of finite wide barrier.

single diffraction process, each of which can be determined by Eq. (1). And $r^{\prime}$ and $r$ represents the distance of $S_{2} P$ and $S_{2} X Y P$, respectively, and -7 in the last term of Eq. (2) is a correction value derived experimentally from Fig. 3 at $N=0(\delta=0)$. Figure 5 shows a comparison between the theoretically predicted values by use of Eq. (2) and experimentally observed values. The horizontal axis shows the theoretical value and the vertical axis shows the experimental value for the finite wide barrier. Therefore, the agreement between them is considered by the deviation of experimental sample values from a $\pi / 4$ straight line passing through the origin. We can observe a good agreement between theory and experiment with the correlation coefficient of 0.945 . These facts indicate that Eq. (2) together with Eq. (1) is extremely available for prediction of the underwater sound attenuation by barrier, including the double diffraction process for wide barrier.

\section{CONCLUSION}

Sound attenuation characteristics of the thin and finite wide barriers for underwater sound propagation were experimentally investigated. Results obtained in the present study were almost the same as in the air. An usefull expression was proposed for the prediction of sound attenuation value and their effectiveness was confirmed. It is believed that our results are very helpful to creat the acoustic transmission system in underwater in the future.

\section{ACKNOWLEDGEMENTS}

We would like to express our cordial thanks to
Y. Honda, Y. Naganawa, K. Shiina and M. Kobayashi for their helpful assistance.

\section{REFERENCES}

1) R. J. Urick, Principles of Under Water Sound (McGraw-Hill, New York, 1967).

2) K. Oimatsu, S. Yamaguchi, S. Kuwahara, and K. Kuramoto, "An experimental consideration of underwater random noise with audio frequency bandwidth in a shallow water area," Acoust. Lett. 13(9), 157-161 (1990).

3) Z. Maekawa, "Noise reduction by screens," Appl. Acoust. 1, 157-173 (1968).

4) K. Yamamoto and K. Takagi, "Expressions of Maekawa's chart for computation," Appl. Acoust. 37, 75-82 (1992).

5) U. J. Kurze, "Noise reduction by barrier," J. Acoust. Soc. Am. 55, 504-518 (1974).

\section{APPENDIX}

In order to prove the fact that the polyethylene mat is useful for eliminating the transmission waves through the barrier, a supplementary experiment was made. Figure A-1 shows the sound pressure waves measured at receiver for the two cases as follows,

(1) Barrier with no polyethylene mat,

(2) Barrier with polyethylene mat.

In experiment, the sound source $\mathbf{S}$ and receiver $\mathbf{P}$ were set up $18(\mathrm{~cm})$ in distance beyond the barrier and were fixed $20(\mathrm{~cm})$ in depth under the surface of water. In the case of the barrier with no polyethylene mat, transmission waves through the barrier ( $\mathrm{A}$ is the propagated wave directly in under-

(1)

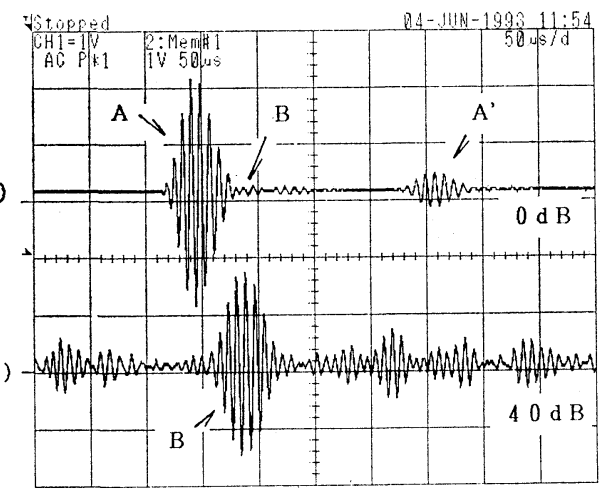

Fig. A-1 Sound pressure waves measured at receiver for the two cases. (1) Barrier with no polyethylene mat. (2) Barrier with polyethylene mat. 


\section{K. KURAMOTO et al.: SOUND ATTENUATION BY BARRIERS IN UNDERWATER}

water and $\mathrm{A}^{\prime}$ is the reflected wave at the water surface) clearly appeared. Here, $B$ is the diffracted wave by the barrier which is much smaller than the transmission waves. On the other hand, in the case of the barrier with polyethylene mat, transmission waves (both $\mathrm{A}$ and $\mathrm{A}^{\prime}$ ) diminished and the diffracted wave B mainly appeared. Of course, each sound wave disappeared when the corresponding sound propagation path was interrupted by a insulation substance. Therefore, the transmission waves through the barrier are almost eliminated by the polyethylene mat and the diffracted wave can be extracted. 